CARPATHIAN J. MATH.

Volume 38 (2022), No. 1,

Pages 217 - 222
Online version at https : //www . carpathian. cunbm . utcluj. ro/

Print Edition: ISSN 1584 - 2851; Online Edition: ISSN 1843 - 4401

DOI: https://doi.org/10.37193/CJM.2022.01.17

Dedicated to the memory of Academician Mitrofan M. Choban (1942-2021)

\title{
Center problem for cubic differential systems with the line at infinity of multiplicity four
}

\author{
AlEXANDRU ŞUBĂ
}

ABSTRACT. In this paper the center problem for cubic differential systems with the line at infinity of multiplicity four is solved.

\section{INTRODUCTION}

Consider the real cubic system of differential equations

$$
\left\{\begin{array}{l}
\dot{x}=y+a x^{2}+c x y+f y^{2}+k x^{3}+m x^{2} y+p x y^{2}+r y^{3} \equiv p(x, y), \\
\dot{y}=-\left(x+g x^{2}+d x y+b y^{2}+s x^{3}+q x^{2} y+n x y^{2}+l y^{3}\right) \equiv q(x, y), \\
\operatorname{gcd}(p, q)=1,(k, l, m, n, p, q, r, s) \neq 0 .
\end{array}\right.
$$

The critical point $(0,0)$ of the system $(1.1)$ is either a focus or a center. The problem of distinguishing between a center and a focus is called the center problem. It is well known that $(0,0)$ is a center if and only if the Lyapunov quantities $L_{1}, L_{2}, \ldots, L_{j}, \ldots$ vanish (see, for example, [2], [6], [7], [8]). Also, the critical point $(0,0)$ is a center if the system (1.1) has an axis of symmetry ([7]) or an analytical integrating factor in a neighborhood of $(0,0)$.

We suppose that the infinity is non-degenerate for (1.1), i.e.

$$
s x^{4}+(k+q) x^{3} y+(m+n) x^{2} y^{2}+(l+p) x y^{3}+r y^{4} \not \equiv 0 .
$$

The homogeneous system associated to the system (1.1) has the form

$$
\left\{\begin{array}{l}
\dot{x}=y Z^{2}+\left(a x^{2}+c x y+f y^{2}\right) Z+k x^{3}+m x^{2} y+p x y^{2}+r y^{3} \equiv P(x, y, Z), \\
\dot{y}=-\left(x Z^{2}+\left(g x^{2}+d x y+b y^{2}\right) Z+s x^{3}+q x^{2} y+n x y^{2}+l y^{3}\right) \equiv Q(x, y, Z) .
\end{array}\right.
$$

Denote $\mathbb{X}=p(x, y) \frac{\partial}{\partial x}+q(x, y) \frac{\partial}{\partial y}, \mathbb{X}_{\infty}=P(x, y, Z) \frac{\partial}{\partial x}+Q(x, y, Z) \frac{\partial}{\partial y}$ and $E_{\infty}=P$. $\mathbb{X}_{\infty}(Q)-Q \cdot \mathbb{X}_{\infty}(P)$. The polynomial $E_{\infty}$ has the form $E_{\infty}=C_{2}(x, y)+C_{3}(x, y) Z+$ $C_{4}(x, y) Z^{2}+\cdots+C_{8}(x, y) Z^{6}$, where $C_{j}(x, y), j=2, \ldots, 8$, are polynomial in $x$ and $y$. We say that the line at infinity $Z=0$ has multiplicity $\nu$ if $C_{2}(x, y) \equiv 0, \ldots, C_{\nu}(x, y) \equiv$ $0, C_{\nu+1}(x, y) \not \equiv 0$, i.e. $\nu-1$ is the greatest positive integer such that $Z^{\nu-1}$ divides $E_{\infty}$. In particular, $Z=0$ has multiplicity four if the identity in $Z$ :

$$
C_{2}(x, y)+C_{3}(x, y) Z+C_{4}(x, y) Z^{2} \equiv 0
$$

holds, i.e. $C_{2}(x, y) \equiv 0, C_{3}(x, y) \equiv 0$ and $C_{4}(x, y) \equiv 0$. If $C_{2}(x, y) \not \equiv 0$, then we say that $Z=0$ has the multiplicity one.

The algebraic line $f(x, y)=0$ is called invariant for (1.1) if there exists a polynomial $K \in \mathbb{C}[x, y]$ such that the identity $\mathbb{X}(f) \equiv f \cdot K(x, y)$ holds. Some notions on multiplicity (algebraic, integrable, infinitesimal, geometric) of an invariant algebraic line and its equivalence for polynomial differential systems are given in [1].

Received: 25.09.2021. In revised form: 04.11.2021. Accepted: 11.04.2021

2010 Mathematics Subject Classification. 34C05.

Key words and phrases. cubic differential system, multiple invariant line, center problem. 
The cubic differential systems with multiple invariant straight lines (including the line at infinity) was studied in [5], [11], [14], and the center problem for (1.1) with invariant straight lines was considered in [2], [3], [4], [9], [10], [12], [13].

In this paper the main result is following:

Theorem 1.1. The cubic system (1.1) with the line at infinity of multiplicity four has at the origin a center if and only if the first three Lyapunov quantities vanish $L_{1}=L_{2}=L_{3}=0$.

\section{ClASSIFICATION OF CUBIC SYSTEMS WITH MULTIPLE LINE AT INFINITY}

Let $\mathcal{X}=(x, y), \mathcal{A}_{2}=(a, b, c, d, f, g), \mathcal{A}_{3}=(k, l, m, n, p, q, r, s), \mathcal{B}_{2}=(A, B, C, D, F, G)$, $\mathcal{U}=(u, v), \mathcal{B}_{3}=(K, L, M, N, P, Q, R, S)$ and $\mathcal{X}=2^{-1} M_{1} \mathcal{U}, \mathcal{A}_{2}=2^{-3} \mathcal{M}_{2} \mathcal{B}_{2}, \mathcal{A}_{3}=$ $2^{-4} \mathcal{M}_{3} \mathcal{B}_{3}$, where

$$
\begin{aligned}
& \mathcal{M}_{1}=\left(\begin{array}{cc}
1 & 1 \\
i & -i
\end{array}\right), \mathcal{M}_{2}=\left(\begin{array}{cccccc}
-i & i & -i & i & -i & i \\
-1 & -1 & 1 & 1 & -1 & -1 \\
-2 & -2 & 0 & 0 & 2 & 2 \\
-2 i & 2 i & 0 & 0 & 2 i & -2 i \\
i & -i & -i & i & i & -i \\
1 & 1 & 1 & 1 & 1 & 1
\end{array}\right), \\
& \mathcal{M}_{3}=\left(\begin{array}{cccccccc}
-i & i & -i & i & -i & i & -i & i \\
i & -i & -i & i & i & -i & -i & i \\
-3 & -3 & -1 & -1 & 1 & 1 & 3 & 3 \\
-3 & -3 & 1 & 1 & 1 & 1 & -3 & -3 \\
3 i & -3 i & -i & i & -i & i & 3 i & -3 i \\
-3 i & 3 i & -i & i & i & -i & 3 i & -3 i \\
1 & 1 & -1 & -1 & 1 & 1 & -1 & -1 \\
1 & 1 & 1 & 1 & 1 & 1 & 1 & 1
\end{array}\right),
\end{aligned}
$$

$\operatorname{det} \mathcal{M}_{1}=-2 i, \operatorname{det} \mathcal{M}_{2}=-2^{9} i, \operatorname{det} \mathcal{M}_{3}=2^{16}, i^{2}=-1$.

We remark that, in general, the elements of $\mathcal{U}, \mathcal{B}_{2}, \mathcal{B}_{3}$ are complex and $v=\bar{u}, B=$ $\bar{A}, D=\bar{C}, G=\bar{F}, L=\bar{K}, N=\bar{M}, Q=\bar{P}, S=\bar{R}$.

In $u, v, A, B, \ldots, R, S$ the non-identity (1.2) and the identity (1.3), up to a non zero factor, look as

$$
C_{3}(u, v)=K u^{4}+(M+S) u^{3} v+(P+Q) u^{2} v^{2}+(N+R) u v^{3}+L v^{4} \not \equiv 0
$$

and

$$
M_{2}(u, v)+M_{3}(u, v) Z+M_{4}(u, v) Z^{2} \equiv 0,
$$

respectively, where

$$
\begin{aligned}
& M_{2}(u, v)=2^{-10} C_{3}(u, v)\left(N_{2}(u, v)+\overline{N_{2}(u, v)}\right), M_{j}(u, v)=2^{j-12}\left(N_{j}(u, v)+\overline{N_{j}(u, v)}\right), j=3,4, \\
& N_{2}(u, v)=(K Q-M S) u^{4}+2(K N-P S) u^{3} v+(3 K L+M N-P Q-3 R S) u^{2} v^{2} / 2 \\
& N_{3}(u, v)=(K(D K+2 A Q-C S)-G K(M-S)-A S(M+S)) u^{7}+(M(D K-G M) \\
& +Q(C K+A M)-S(G M+A Q)+2 K(B K+2 A N+G(Q-P)+S(D-F)) \\
& -2 S(A P+C(M+S))) u^{6} v+3((M(A N-G P)+K(2 A L+B M+C N+G N+D Q \\
& -G R+B S)-S(F M+C P+G P+C Q+A R+F S))) u^{5} v^{2}+(2 P(A N-G P) \\
& +Q(D M+A N-C P-2 G P-C Q-A R)+K(5 C L+4 G L+4 D N+2 F N+2 B P \\
& +4 B Q-D R)+M(5 A L+B M+2 C N+G N-D P-F Q-4 G R)+S(A L+B M \\
& -2 C N-2 D P-4 F P-5 F Q-4 C R-5 G R)) u^{4} v^{3} ;
\end{aligned}
$$


$N_{4}(u, v)=\left(A^{2} Q+K(G(G-C)-2 M+2 S)+A(2 D K-C S-G(M+S))\right) u^{6}$ $+(A(D M+2 A N-2 G P+C Q)-2 M(C G+M)+K(4 A B+C D+3 D G-2 F G-4 P$ $\left.+8 Q)-S\left(C^{2}+2 A F+3 C G+4 M+6 S\right)\right) u^{5} v+(B(3 C+4 G) K+D(2 D K+G M)$ $-G^{2} P-2 M(3 P-Q)+2 K(7 N-3 R)-10 S(P+Q)-F G(3 M+5 S)+A(3 A L+3 B M$ $+3 C N+G N+D Q-3 G R+B S)-C(3 G P+2 G Q+2 D S+3 F S)) u^{4} v^{2}$

$+(10 K L-2 P(2 P+Q)+4 M(N-2 R)+A(5 C L+2 G L+2 D N+2 F N+B P$ $+B Q-D R)+C(C N-G N-D P-F Q-4 G R)-8 R S-2 F(2 G P+F S)) u^{3} v^{3}$.

The identity $N_{2}(u, v)+\overline{N_{2}(u, v)} \equiv 0$ gives us the following three set of conditions:

$$
\begin{gathered}
K=L=R=S=0, P=\alpha M, Q=N / \alpha, M N \neq 0, \alpha \in \mathbb{C}, \alpha \bar{\alpha}=1 \\
M=N=P=Q=0, R=\beta K, S=L / \beta, K L \neq 0, \beta \in \mathbb{C}, \beta \bar{\beta}=1 \\
P=\gamma N, Q=M / \gamma, R=\gamma L, S=K / \gamma, K L M N \neq 0, \gamma \in \mathbb{C}, \gamma \bar{\gamma}=1 .
\end{gathered}
$$

Lemma 2.1. The line at infinity has the multiplicity at least two for cubic system $\{(1.1),(1.2)\}$ if and only if the coefficients of $\{(1.1),(1.2)\}$ verify one of the set of conditions (2.4), (2.5) and (2.6).

Under the conditions (2.4), (2.5) and (2.6) we have respectively:

$$
\begin{aligned}
M_{3}(u, v)= & -u v(u+\alpha v)\left((F N-\alpha B M)\left(2 N u v+\alpha M u^{2}+\alpha N v^{2}\right) v^{2}+(C N-\alpha D M) .\right. \\
& \left.\left(N-\alpha^{2} M\right) u^{2} v^{2}-\alpha(A N-\alpha G M)\left(M u^{2}+N v^{2}+2 \alpha M u v\right) u^{2}\right) / \alpha^{2} \equiv 0 \Rightarrow
\end{aligned}
$$

$$
K=L=R=S=0, F=B / \alpha, G=\alpha A, N=\alpha^{2} M, P=\alpha M, Q=\alpha M, M \neq 0, \alpha \bar{\alpha}=1 ;
$$

$$
\begin{aligned}
& K=L=R=S=0, D=C N /(\alpha M), F=\alpha B M / N, G=A N /(\alpha M), \\
& P=\alpha M, Q=N / \alpha, M\left(N-\alpha^{2} M\right) \neq 0, \alpha \bar{\alpha}=1 ;
\end{aligned}
$$$$
M_{3}(u, v)=-\left(u^{3}+\beta v^{3}\right)\left(v ( 2 u ^ { 3 } - \beta v ^ { 3 } ) \left(C L^{2}-\beta D K L+\beta F K L-\beta^{2} B K^{2}\right.\right.
$$$$
+u\left(u^{3}-2 \beta v^{3}\right) \cdot\left(A L^{2}+\beta C K L-\beta G K L-\beta^{2} D K^{2}\right)
$$$$
\left.+3 u^{2} v^{2}\left(F L^{2}-\beta B K L-\beta^{2} A K L+\beta^{3} G K^{2}\right)\right) / \beta^{2} \equiv 0 \Rightarrow
$$

$$
\begin{aligned}
& \quad M=N=P=Q=0, F=D+\left(\beta^{2} B K^{2}-C L^{2}\right) /(\beta K L), \\
& G=C+\left(A L^{2}-\beta^{2} D K^{2}\right) /(\beta K L), R=\beta K, S=L / \beta, L^{3}-\beta^{4} K^{3}=0, \beta \bar{\beta}=1 ; \\
& M_{3}(u, v)=\left(K u^{3}+M u^{2} v+\gamma N u v^{2}+\gamma L v^{3}\right)\left((\gamma K(\gamma D-C)-(A-\gamma G)(K-\gamma M)) u^{4}\right. \\
& -2\left(\gamma^{2} N(\gamma G-A)+K\left(C-\gamma D+\gamma F-\gamma^{2} B\right)\right) u^{3} v+(\gamma(A-\gamma G)(N+3 \gamma L) \\
& \left.-(F-\gamma B)(3 K+\gamma M)-(C-\gamma D)\left(M-\gamma^{2} N\right)\right) u^{2} v^{2}-2(M(F-\gamma B)-\gamma L(A \\
& \left.\left.\left.+\gamma C-\gamma G-\gamma^{2} D\right)\right) u v^{3}+\gamma(C L-\gamma D L-(F-\gamma B)(N-\gamma L)) v^{4}\right) / \gamma^{2} \equiv 0 \Rightarrow
\end{aligned}
$$

$$
\begin{aligned}
& M=N=P=Q=0, C=\beta D K / L, F=\beta B K / L, \\
& G=A L /(\beta K), R=\beta K, S=L / \beta, \beta \bar{\beta}=1 ;
\end{aligned}
$$

$$
\begin{gathered}
D=C / \gamma, F=B \gamma, G=A / \gamma, P=\gamma N, R=\gamma L, \\
Q=M / \gamma, S=K / \gamma, K M \neq 0, \gamma \bar{\gamma}=1 \\
D=\left(C L \gamma^{3}+(F-B \gamma)(K-M \gamma)\right) /\left(L \gamma^{4}\right), G=\left(K(B \gamma-F)+A L \gamma^{2}\right) /\left(L \gamma^{3}\right), \\
N=\left(-K+M \gamma+L \gamma^{4}\right) / \gamma^{3}, P=\left(-K+M \gamma+L \gamma^{4}\right) / \gamma^{2}, R=L \gamma, \\
Q=M / \gamma, S=K / \gamma, M(F-B \gamma) \neq 0, \gamma \bar{\gamma}=1 .
\end{gathered}
$$

Substituting (2.7), (2.8) and (2.9) in the polynomial $M_{4}(u, v)$ we obtain, respectively,

$$
\begin{aligned}
M_{4}(u, v)= & M u v\left(u^{3} \alpha(A D-2 M-A C \alpha)(u+2 v \alpha)-v^{3} \alpha(2 u+v \alpha)\left(B D-B C \alpha+2 M \alpha^{3}\right)\right. \\
& -u^{2} v^{2}\left(4 M \alpha^{3}+(D-C \alpha)\left(B-D \alpha+C \alpha^{2}-A \alpha^{3}\right)\right) / \alpha \not \equiv 0 ; \\
M_{4}(u, v)= & -2 u v(u+v \alpha)\left(N^{2} v^{3} \alpha+N u v^{2}\left(2 N-M \alpha^{2}\right)-M u^{2} v \alpha\left(N-2 M \alpha^{2}\right)\right. \\
& \left.+M^{2} u^{3} \alpha^{2}\right) / \alpha^{2} \not \equiv 0 \\
M_{4}(u, v)= & 2\left(u^{3}+v^{3} \beta\right)\left(K L u^{3} \beta-3 L^{2} u^{2} v-3 K^{2} u v^{2} \beta^{3}+K L v^{3} \beta^{2}\right) / \beta^{2} \not \equiv 0 .
\end{aligned}
$$


Similarly, it can be verified that the polynomial $M_{4}(u, v)$ is not identical zero in the case (2.10). For this it is sufficient to examine separately the identity $M_{4}(u, v) \equiv 0$ in cases $D L^{2}-C K^{2} \beta^{3}=0$ and $D L^{2}-C K^{2} \beta^{3} \neq 0$.

In this way we have proved the following Lemma.

Lemma 2.2. The line at infinity has the multiplicity at least three for cubic system $\{(1.1),(1.2)\}$ if and only if the coefficients of $\{(1.1),(1.2)\}$ verify one of the set of conditions (2.7) - (2.12). In the cases $(2.7)-(2.10)$ the multiplicity is exactly three.

To obtain the cubic systems (1.1) which have the line at infinity of multiplicity four we will investigate the identity $M_{4}(u, v) \equiv 0$ in each of the series of conditions (2.11) and (2.12):

$$
\begin{aligned}
\left.M_{4}(u, v)\right|_{(2.11)}= & 2\left(K u^{3}+M u^{2} v+N u v^{2} \gamma+L v^{3} \gamma\right)\left(u^{3} \gamma(K-M \gamma)-u^{2} v(3 K-M \gamma\right. \\
& \left.\left.+2 N \gamma^{3}\right)-u v^{2}\left(2 M-N \gamma^{2}+3 L \gamma^{3}\right)-v^{3} \gamma(N-L \gamma)\right) / \gamma^{2} \equiv 0 \Rightarrow \\
& D=C S / K, F=B K / S, G=A S / K, L=-S^{4} / K^{3} \\
& M=S, N=R=-S^{3} / K^{2}, Q=-P=S^{2} / K
\end{aligned}
$$

$$
\begin{aligned}
& \left.M_{4}(u, v)\right|_{(2.12)}=\left(K u^{2} \gamma-K u v+M u v \gamma+L v^{2} \gamma^{3}\right)\left(u ^ { 4 } \gamma ^ { 2 } \left(K^{2}(F-B \gamma)^{2}+\left(A K L \gamma^{2}\right.\right.\right. \\
& \left.\left.\quad+C K L \gamma^{3}-A L M \gamma^{3}\right)(F-B \gamma)+2 L^{2} \gamma^{5}(K-M \gamma)\right)-2 u^{3} v \gamma\left(2 L^{2} \gamma^{6}\left(M+L \gamma^{3}\right)\right. \\
& \left.\quad+(F-B \gamma)\left(K(F-B \gamma)(K-M \gamma)-K L \gamma^{3}(C+F \gamma)-A L \gamma^{2}\left(K-M \gamma-L \gamma^{4}\right)\right)\right) \\
& \quad+u^{2} v^{2}\left(\left(K^{2}-2 K M \gamma+M^{2} \gamma^{2}+5 K L \gamma^{4}+L M \gamma^{5}\right)(F-B \gamma)^{2}+L \gamma^{2}(A K+C K \gamma\right. \\
& \left.\left.\quad-A M \gamma+3 B K \gamma^{3}-4 A L \gamma^{4}+B M \gamma^{4}-C L \gamma^{5}\right)(F-B \gamma)-4 L^{2} \gamma^{5}\left(K+M \gamma+2 L \gamma^{4}\right)\right) \\
& \quad-2 L u v^{3} \gamma^{3}\left(\left(F K-B K \gamma-2 F M \gamma+A L \gamma^{2}+B M \gamma^{2}+C L \gamma^{3}\right)(F-B \gamma)+2 L \gamma^{2}(M\right. \\
& \left.\left.\quad+L \gamma^{3}\right)\right)-L v^{4} \gamma^{2}\left(\left(F K-F M \gamma+C L \gamma^{3}-F L \gamma^{4}+B L \gamma^{5}\right)(F-B \gamma)\right. \\
& \quad-2 L \gamma(K-M \gamma))) /\left(L^{2} \gamma^{9}\right) \equiv 0 \Rightarrow
\end{aligned}
$$

$$
\begin{aligned}
& A=2\left(K^{3} L+S^{4}\right) /\left(S^{2}(B K-F S)\right)-S(B K-2 F S) /(K L), R=K L / S, \\
& C=2\left(K^{3} L+S^{4}\right) /(K S(B K-F S))-\left(B K^{4} L-2 F K^{3} L S-F S^{5}\right) /\left(K^{2} L S^{2}\right), \\
& D=\left(F K^{2} L+B S^{3}\right) /\left(K^{2} L\right)+2\left(K^{3} L+S^{4}\right) /\left(K^{2}(B K-F S)\right), \\
& G=F S^{3} /\left(K^{2} L\right)+2\left(K^{3} L+S^{4}\right) /(K S(B K-F S)), M=\left(K^{3} L+2 S^{4}\right) / S^{3} \\
& N=\left(2 K^{3} L+S^{4}\right) /\left(K^{2} S\right), P=\left(2 K^{3} L+S^{4}\right) /\left(K S^{2}\right), Q=\left(K^{3} L+2 S^{4}\right) /\left(K S^{2}\right) .
\end{aligned}
$$

Lemma 2.3. The line at infinity has the multiplicity at least four for cubic system $\{(1.1),(1.2)\}$ if and only if the coefficients of $\{(1.1),(1.2)\}$ verify one of the set of conditions (2.13) and (2.14).

\section{PROOF OF THE THEOREM 1.1}

Lemma 3.4. The following four sets of conditions are sufficient conditions for the origin $(0,0)$ to be a center for system (1.1):

$$
\begin{aligned}
& C=4 K S^{3} /\left(A S^{3}-B K^{3}\right), D=4 S^{4} /\left(A S^{3}-B K^{3}\right), F=B K / S, G=A S / K, M=S, \\
& L=-S^{4} / K^{3}, N=R=-S^{3} / K^{2}, Q=-P=S^{2} / K, B^{2} K^{6}+4 K^{2} S^{5}-A^{2} S^{6}=0 \\
& A=2\left(K^{3} L+S^{4}\right) /\left(S^{2}(B K-F S)\right)-S(B K-2 F S) /(K L), R=K L / S, \\
& C=2\left(K^{3} L+S^{4}\right) /(K S(B K-F S))-\left(B K^{4} L-2 F K^{3} L S-F S^{5}\right) /\left(K^{2} L S^{2}\right), \\
& D=\left(F K^{2} L+B S^{3}\right) /\left(K^{2} L\right)+2\left(K^{3} L+S^{4}\right) /\left(K^{2}(B K-F S)\right), \\
& G=F S^{3} /\left(K^{2} L\right)+2\left(K^{3} L+S^{4}\right) /(K S(B K-F S)), M=\left(K^{3} L+2 S^{4}\right) / S^{3}, \\
& N=\left(2 K^{3} L+S^{4}\right) /\left(K^{2} S\right), P=\left(2 K^{3} L+S^{4}\right) /\left(K S^{2}\right), Q=\left(K^{3} L+2 S^{4}\right) /\left(K S^{2}\right), \\
& \left(B K^{6} L^{2}-2 F K^{5} L^{2} S+B S^{8}\right)(B K-F S)+2 L S\left(K^{3} L+S^{4}\right)^{2}=0
\end{aligned}
$$




$$
\begin{aligned}
& A=\left(2 S^{3}-F^{2} K^{2}\right) /\left(F S^{2}\right), B=S\left(F^{2} K^{2}-2 S^{3}\right) /\left(F K^{3}\right), C=2 S^{2} /(F K), \\
& D=-2 S^{3} /\left(F K^{2}\right), G=-F K / S, L=S^{4} / K^{3}, M=3 S, N=3 S^{3} / K^{2}, \\
& P=3 S^{2} / K, Q=3 S^{2} / K, R=S^{3} / K^{2} ; \\
& A=\left(2 S^{3}-F^{2} K^{2}\right) /\left(F S^{2}\right), B=S\left(F^{2} K^{2}+2 S^{3}\right) /\left(F K^{3}\right), C=-2 S^{2} /(F K), \\
& D=-2 S^{3} /\left(F K^{2}\right), G=-F K / S, L=-S^{4} / K^{3}, M=S, N=-S^{3} / K^{2}, \\
& P=-S^{2} / K, Q=S^{2} / K, R=-S^{3} / K^{2} .
\end{aligned}
$$

Proof. When one of the condition (3.15), (3.16) holds the system (1.1) has an affine invariant straight line $l_{1}$ and a Darboux integrating factor of the form $\mu(x, y)=1 / l_{1}$.

In the case (3.15): $l_{1}=2 S^{2}\left(B K^{3}-A S^{3}\right)+\left(B^{2} K^{4}-A B K S^{3}+2 S^{5}\right)((S-K) x+i(K+$ S) $y), i^{2}=-1$.

In the case (3.16): $l_{1}=4 K^{2} L S\left(K^{3} L+S^{4}\right)+(B K-F S)\left(K^{3} L-S^{4}\right)\left(\left(K^{2} L+S^{3}\right) x+\right.$ $\left.i\left(K^{2} L-S^{3}\right) y\right), i^{2}=-1$.

Under the conditions (3.17) the equalities $C F-D G=A D^{3}-B C^{3}=A F^{3}-B G^{3}=$ $A^{4} L^{3}-B^{4} K^{3}=A^{2} N^{3}-B^{2} M^{3}=A^{2} R^{3}-B^{2} S^{3}=C^{4} L-D^{4} K=C^{2} N-D^{2} M=$ $C^{2} R-D^{2} S=F^{4} K-G^{4} L=F^{2} M-G^{2} N=F^{2} S-G^{2} R=K N^{2}-L M^{2}=K R^{2}-L S^{2}=$ $M R-N S=P-Q=0$ hold. Therefore, the system $\{(1.1),(3.17)\}$ has an axis of symmetry and the origin is a center ([7]).

In the case (3.18) the system (1.1) has the integrating factor of the Darboux form:

$$
\mu(x, y)=l_{1} l_{2}^{\alpha_{2}} l_{3}^{\alpha_{3}} l_{4}^{\alpha_{4}},
$$

where

$$
\begin{aligned}
& l_{1}= 2 F-\left(S^{2}(K x+S x-i K y+i S y)\right) / K^{2}, \quad l_{2}=\operatorname{Exp}[x+S x / K-i y+i S y / K], \\
& l_{3}= \operatorname{Exp}\left[16 S^{2} x / K^{2}-\left(F(K-S)(K x+S x-i K y+i S y)^{2}\right) / K^{3}\right], \\
& l_{4}= \operatorname{Exp}\left[6 S(K x-S x-i K y-i S y)(K x+S x-i K y+i S y) / K^{3}\right. \\
&\left.\quad+F(K x+S x-i K y+i S y)^{3} / K^{3}\right], i^{2}=-1 \\
& \alpha_{2}=F K(K+S) /(2 S(K-S)), \alpha_{3}=F K^{3} /\left(8 S^{2}(S-K)\right), \alpha_{4}=-K / 24 .
\end{aligned}
$$

To prove the Theorem 1.1, we compute the first three Lyapunov quantities $L_{1}, L_{2}, L_{3}$ for each sets of conditions (2.13) and (2.14). In the expressions for $L_{j}$, we will neglect the non-zero factors.

In the case (2.13) the first Lyapunov quantity is $L_{1}=4 K S^{3}+C\left(B K^{3}-A S^{3}\right)$ and $L_{1}=0$ gives $\left.C=-4 K S^{3}\right) /\left(B K^{3}-A S^{3}\right)$. Substituting the expression of $C$ in $L_{2}$ and $L_{3}$ we obtain $L_{2}=g_{1} g_{2}$ and $L_{3}=g_{1} g_{3}$, where $g_{1}=B^{2} K^{6}+4 K^{2} S^{5}-A^{2} S^{6}, g_{2}=B^{2} K^{6}-16 K^{2} S^{5}-A^{2} S^{6}$ and $g_{3}=\left(61 A B^{3} K^{7}+339 B^{2} K^{6} S^{2}-122 A^{2} B^{2} K^{4} S^{3}+61 A^{3} B K S^{6}+1512 K^{2} S^{7}-339 A^{2} S^{8}\right)$. $g_{2}+4320 K^{4} S^{12}$.

If $g_{1}=0$, then Lemma 3.4, (3.15). Taking into account that $K S \neq 0$, the system of equalities $\left\{g_{2}=0, g_{3}=0\right\}$ do not have solutions.

In the case (2.14) the first Lyapunov quantity is $L_{1}=f_{1} f_{2}$, where $f_{1}=\left(B K^{6} L^{2}-\right.$ $\left.2 F K^{5} L^{2} S+B S^{8}\right)(B K-F S)+2 L S\left(K^{3} L+S^{4}\right)^{2}$ and $f_{2}=B F K+2 K L-F^{2} S$. If $f_{1}=0$, then Lemma 3.4, (3.16). Assume that $f_{1} \neq 0$ and let $f_{2}=0$. Then we find that $B=$ $\left(F^{2} S-2 K L\right) /(F K)$ and $L_{2}=\left(K^{3} L-S^{4}\right)\left(K^{3} L+S^{4}\right)$. If $K^{3} L-S^{4}=0$, then Lemma 3.4, (3.17), and if $K^{3} L+S^{4}=0$, then Lemma 3.4, (3.18). Theorem 1.1 is proved.

From the proof of Theorem 1.1 it results the following statement:

Theorem 3.2. The cubic system (1.1) with the line at infinity of multiplicity four has a center at the origin if and only if the coefficients of (1.1) verify one of the sets of conditions (3.15)-(3.18).

Example 3.1. Consider the cubic system

$$
\dot{x}=y, \quad \dot{y}=\left(-4 x+3 x^{2}+4 x y+2 x^{2} y-5 y^{2}\right) / 4 .
$$


For this system the line at infinity has multiplicity four: $E_{\infty}=-Z^{3}\left(2 x^{2} y\left(3 x^{2}+5 y^{2}\right)+\left(9 x^{4}+\right.\right.$ $\left.\left.4 x^{3} y+4 x y^{3}-25 y^{4}\right) Z-8(3 x+2 y)\left(x^{2}+y^{2}\right) Z^{2}+16\left(x^{2}+y^{2}\right) Z^{3}\right) / 16$. The first two Lyapunov quantities vanish $\left(L_{1}=L_{2}=0\right)$ and the third one is $L_{3}=75 / 1024 \neq 0$. Therefore, the origin is a focus of multiplicity three.

\section{REFERENCES}

[1] Christopher, C.; Llibre, J.; Pereira, J. V. Multiplicity of invariant algebraic curves in polynomial vector fields. Pacific J. Math. 329 (2007), no. 1, 63-117.

[2] Cozma, D. Integrability of cubic systems with invariant straight lines and invariant conics. Î. E. P. Ştiinţa, Chişinău, 2013.

[3] Cozma, D.; Şubă, A. The solution of the problem of center for cubic differential systems with four invariant straight lines. Analele Ştiinţifice ale Universităţii "Al. I. Cuza", Iaşi, s.I.a, Matematică, 44 (1998), 517-530.

[4] Cozma, D.; Şubă, A. Solution of the problem of the centre for a cubic differential system with three invariant straight lines. Qual. Theory Dyn. Syst. Universitat de Lleida. Spain. 2 (2001), no. 1, 129-143.

[5] Llibre, J.; Vulpe, N. Planar cubic polynomial differential systems with the maximum number of invariant straight lines. Rocky Mountain J. Math. 36 (2006), no. 4, 1301-1373.

[6] Romanovski, V. G.; Shafer, D. S. The center and cyclicity problems: a computational algebra approach. Boston, Basel, Berlin : Birkhäuser, 2009.

[7] Sibirsky, C. S. Algebraical invariants of differential equations and matrices. Kishinev, Shtiintsa, 1976 (in Russian).

[8] Şubă, A. On the Lyapunov quantities of two-dimensional autonomous systems of differential equations with a critical point of centre or focus type. Buletin of Baia Mare University (Romany). Mathematics and Informatics. 13 (1998), no. 1-2,153-170.

[9] Şubă, A. The center conditions for a cubic system. Bul. Ştiinţ. Univ. Baia Mare, Ser. B, Mathematică şi Informatică. XVIII (2002), no. 2, 355-360.

[10] Şubă, A.; Cozma, D. Solution of the problem of the center for cubic differential system with three invariant straight lines in generic position. Qualitative Theory of Dynamical Systems, Universitat de Lleida, Spain. 6 (2005), 45-58.

[11] Şubă, A.; Repeşco, V.; Puţuntică, V. Cubic systems with invariant affine straight lines of total parallel multiplicity seven. Electron. J. Diff. Equ., vol. 2013 (2013), no. 274, pp. 1-22. http:/ / ejde.math.txstate.edu/

[12] Şubă, A.; Turuta, S. Solution of the problem of the center for cubic differential systems with the line at infinity and an affine real invariant straight line of total algebraic multiplicity five. Bulletin of Academy of Sciences of the Republic of Moldova. Mathematics. 2019, no. 2 (90), 13-40.

[13] Şubă, A.; Turuta, S. Solution of the center problem for cubic differential systems with one or two affine invariant straight lines of total algebraic multiplicity four. ROMAI J. 15 (2019), no. 2, 101-116.

[14] Şubă, A.; Vacaraş, O. Cubic differential systems with an invariant straight line of maximal multiplicity. Annals of the University of Craiova, Mathematics and Computer Science Series, 42 (2015), no. 2, 427-449.

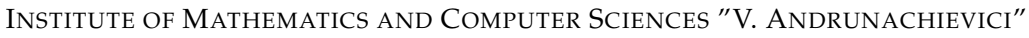

ACADEMIEI 5, MD-2028, CHIŞINĂU, REPUbliC OF MOLdOVA

TIRASPOL STATE UNIVERSITY

GH. IAblociKin 5, MD-2069, Chişinău, Republic OF MOldova

Email address: suba@math.md 